\title{
Fortificação de alimentos com o pó da casca de ovo como fonte de cálcio
}

Food fortification with egg shell powder as a calcium source

\author{
Maria Margareth Veloso NAVES ${ }^{1 *}$, Daniela Canuto FERNANDES ${ }^{1}$, \\ Carla Marques Maia PRADO ${ }^{1}$, Luíz Sávio Medeiros TEIXEIRA ${ }^{2}$
}

\begin{abstract}
Resumo
O pó da casca de ovo é usado como fonte de cálcio em multimisturas distribuídas no Brasil por organizações não-governamentais. O presente trabalho teve por objetivos fortificar, com o pó da casca de ovo, alimentos tradicionais, de baixo custo e de fácil preparo, e estimar a contribuição nutricional de porções desses alimentos para o aporte diário de cálcio. Foram elaboradas dez preparações de consumo habitual, que foram enriquecidas com o pó da casca de ovo $(37,4 \%$ de cálcio) na proporção de $1 \mathrm{~g}$ do pó para 100 g de cereal ou farinha usada nos alimentos processados. Os alimentos fortificados apresentaram teores de cálcio entre 111,5 mg e 506,4 mg. $100 \mathrm{~g}^{-1}$, significativamente mais elevados que o conteúdo das formulações originais sem fortificação. Porções médias das formulações fortificadas perfazem de 14 a $32 \%$ das referências nutricionais de cálcio para indivíduos adultos. Conclui-se que alimentos de consumo habitual fortificados com o pó da casca de ovo podem contribuir de forma significativa para a ingestão adequada de cálcio e prevenção de deficiência do nutriente, especialmente de osteoporose, em indivíduos de diferentes grupos etários e extratos sociais.

Palavras-chave: cálcio na dieta; casca de ovo; alimentos fortificados; osteoporose.
\end{abstract}

\begin{abstract}
Egg shell powder is used as a calcium source in a nutritional supplement known as "multimistura" that has been distributed in Brazil by non-governmental organizations. The objectives of this research were to fortify traditional, low cost and easy to prepare dishes with egg shell powder and to estimate the nutritional contribution of each portion to the calcium daily intake. Ten common dishes were prepared and fortified with egg shell powder (with $37.4 \%$ of calcium) at the rate of $1 \mathrm{~g}$ to $100 \mathrm{~g}$ of cereal or flour used in the processing foods. The fortified foods showed a range of calcium concentrations between $111.5 \mathrm{mg}$ and $506.4 \mathrm{mg} .100 \mathrm{~g}^{-1}$, which were significantly higher than the contents of the original formulations without fortification. Mean portions of fortified dishes provide about 14 to $32 \%$ of the nutritional calcium references for adults. It was concluded that traditional dishes fortified with egg shell powder could contribute significantly to the adequate calcium intake and to prevent calcium deficiency, mainly osteoporosis, in individuals of different age groups and social levels.

Keywords: dietary calcium; egg shell; fortified food; osteoporosis.
\end{abstract}

\section{Introdução}

O cálcio é um mineral de extrema importância para o organismo, sobretudo para a mineralização óssea, sendo que as necessidades deste mineral são relativamente maiores nos períodos de gestação, lactação, adolescência e senescência ${ }^{23,28}$. A ingestão adequada de cálcio, desde os primeiros anos de vida e, em especial, durante a adolescência, garante melhor densidade do osso, retardando assim o processo de perda óssea e subseqüente desenvolvimento de osteoporose ${ }^{6,12,30}$. Esta enfermidade é mais prevalente em mulheres no período pósmenopausa e em idosas, sendo, contudo, observada também entre adolescentes com amenorréia e em indivíduos adultos vegetarianos. O consumo baixo ou nulo de leite e seus derivados, especialmente durante as fases de crescimento, constitui um dos principais fatores que predispõe ao desenvolvimento de osteoporose $\mathrm{e}^{12,18,29,30}$.

Diversas estratégias têm sido propostas a fim de elevar o consumo de cálcio e minimizar os danos causados por sua deficiência. O cálcio pode ser obtido a partir da ingestão de alimentos naturalmente ricos no mineral, tais como leite e produtos derivados, alimentos fortificados ou ainda por meio

Recebido para publicação em 20/3/2006

Aceito para publicação em 24/1/2007 (001693)

Faculdade de Nutrição, Universidade Federal de Goiás,

Rua $227 \mathrm{~s} / n^{\circ}$, quadra 68, Setor Universitário, CEP 74605-080,

Goiânia-GO, Brasil

E-mail:mnaves@fanut.ufg.br

${ }^{2}$ Laboratório Nacional Agropecuário de Goiás - LANAGRO/MAPA,

Goiânia - GO, Brasil

*A quem a correspondência deve ser enviada de suplementos dietéticos ${ }^{28}$. Todavia, a ingestão de cálcio é, em geral, insuficiente para atingir as necessidades do organismo, sobretudo no caso de adolescentes e idosos. Estudos descritivos conduzidos em vários países mostram um consumo de cálcio inadequado, muitas vezes abaixo de $50 \%$ da ingestão preconizada $^{7,13,20}$. Outros fatores contribuem para o agravamento dessa situação de aporte inadequado de cálcio, como a presença de fitatos na dieta e o consumo elevado de proteínas, que podem reduzir a biodisponibilidade do mineral ${ }^{8}$.

A população de baixa renda tem acesso limitado aos alimentos fonte de cálcio, sendo alvo de programas que incentivam o consumo de fontes alternativas desse nutriente. Assim, organizações não-governamentais têm utilizado o pó da casca de ovo como fonte de cálcio de baixo custo. O cálcio presente nesse pó é biodisponível, conforme constatado em experimentos in vivo, tanto em dieta padrão quanto em dieta à base da mistura arroz-feijão ${ }^{15,27}$, o que indica que o produto pode ser uma fonte adequada para suprir as necessidades de cálcio do organismo. Além disso, foi comprovado, mediante análises microbiológicas, que a adoção de procedimentos adequados no preparo do pó minimiza os riscos de contaminação microbiológica e assegura a qualidade do produto ${ }^{16,17}$.

O presente trabalho teve por objetivos fortificar, com o pó da casca de ovo, alimentos de baixo custo e fácil preparo, e estimar a contribuição nutricional de porções dos alimentos formulados para o aporte diário de cálcio. 


\section{Material e métodos}

\subsection{Preparo do pó da casca de ovo}

Foram usadas cascas de ovos brancos de galinha, adquiridas em um estabelecimento comercial da cidade de Goiânia. A partir da forma de preparo do pó utilizada pela Pastoral da Criança, padronizou-se uma técnica de higienização das cascas, conforme os parâmetros preconizados pela INTERNATIONAL COMMISSION ON MICROBIOLOGICAL SPECIFICATION FOR FOODS ${ }^{11}$ e por SILVA JUNIOR ${ }^{26}$. As cascas foram submetidas à seguinte técnica de higienização: lavagem em água corrente, imersão em solução de hipoclorito de sódio por 10 minutos e fervura durante 10 minutos. Após esse procedimento, as cascas foram secas em estufa a $50{ }^{\circ} \mathrm{C}$ (FANEM, modelo $315 \mathrm{SE}$ ) por 2 horas, trituradas em liquidificador (WALLITA, Beta) e peneiradas através de um pano fino, obtendo-se o pó da casca de ovo.

\subsection{Elaboração das formulações fortificadas com pó da casca de ovo}

Foram testadas dez formulações de baixo custo e de fácil preparo consumidas habitualmente na região, conforme apresentado na Tabela 1. Os alimentos foram fortificados com o pó da casca de ovo, na proporção de aproximadamente $1 \mathrm{~g}$ do pó para $100 \mathrm{~g}$ de cereal ou farinha utilizada no preparo dos pratos. A quantidade de pó da casca de ovo adicionada às formulações foi definida visando alcançar boa concentração de cálcio no alimento, sem comprometer sua aceitação. Para tanto, após o preparo, procedeu-se a uma avaliação preliminar destas fórmulas com teste de aceitação informal com cerca de oito participantes. Além disso, foram estabelecidas as porções médias de cada formulação baseando-se em estudo prévio com alimentos regionais ${ }^{22}$.

\subsection{Determinação da composição centesimal das formulações fortificadas}

As amostras dos alimentos processados foram submetidas, em três repetições, às análises descritas a seguir. A determinação da umidade foi realizada por secagem da amostra em estufa a $105^{\circ} \mathrm{C}$ até peso constante, conforme descrição do INSTITUTO ADOLFO LUTZ ${ }^{9}$. O teor de nitrogênio foi determinado pelo método de Kjeldahl, segundo $\mathrm{AOAC}^{1}$ e convertido em proteína bruta utilizando-se os fatores correspondentes para cada tipo de alimento, ou o fator 6,25 para as preparações que continham mais de um alimento fonte de proteína. Os lipídios totais foram extraídos segundo a técnica descrita por BLIGH \& DYER ${ }^{2}$ e, posteriormente, determinados por gravimetria. O resíduo mineral fixo foi analisado utilizando-se o método de incineração a $550{ }^{\circ} \mathrm{C}$, de acordo com $\mathrm{AOAC}^{1}$. Os carboidratos totais foram determinados pelo cálculo da diferença entre $100 \mathrm{~g}$ do alimento e a soma total dos valores encontrados para umidade, proteína, lipídios e resíduo mineral fixo. Neste cálculo, o teor de fibra alimentar não foi considerado separadamente, pois as formulações estudadas não constituem fonte do nutriente ${ }^{24}$. O valor energético total dos alimentos foi estimado considerando-se

Tabela 1. Ingredientes (tipo e quantidade) usados nas formulações fortificadas com o pó da casca de ovo.

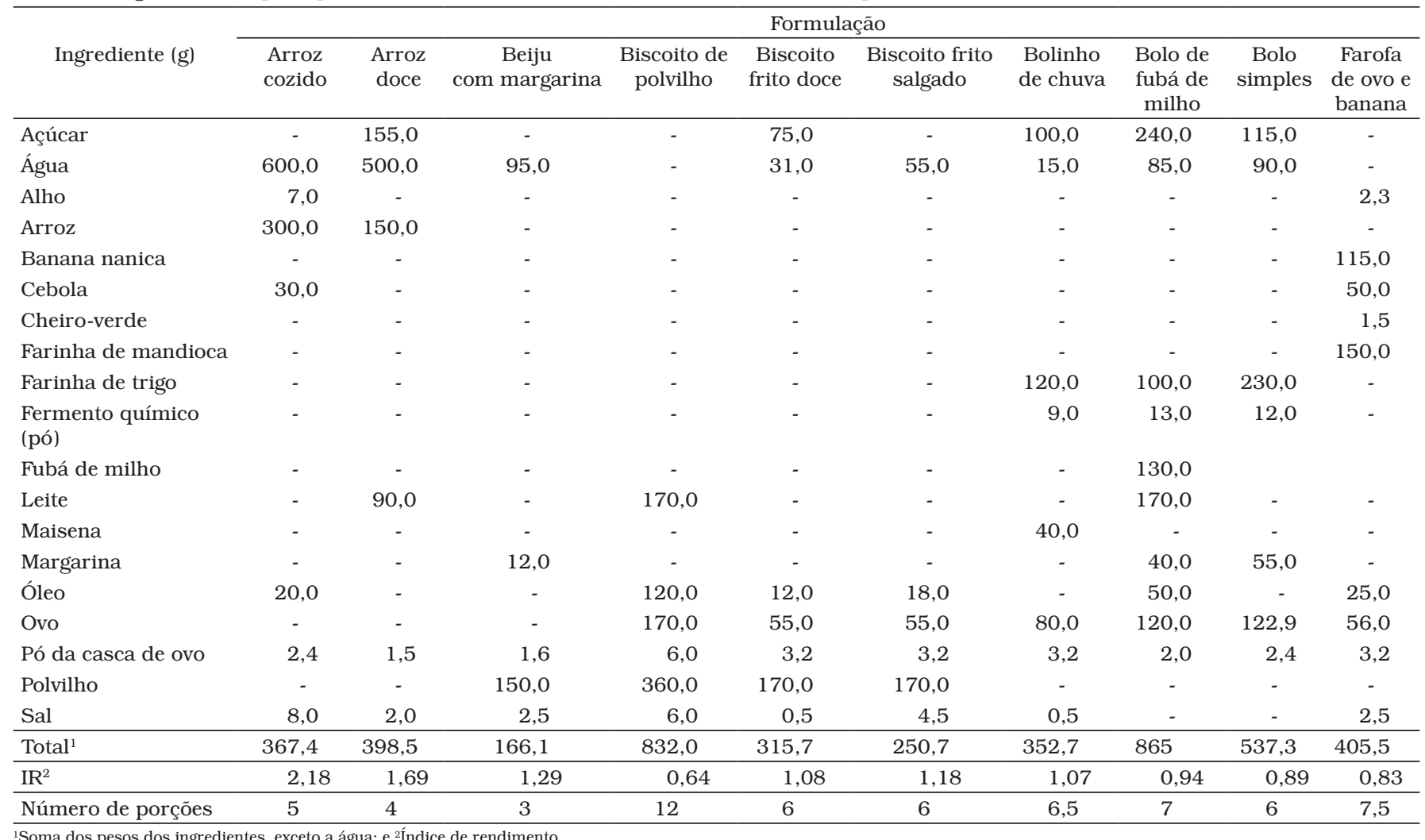


os fatores de conversão de Atwater de $4 \mathrm{kcal} . \mathrm{g}^{-1}$ de proteína, 4 kcal.g ${ }^{-1}$ de carboidrato e 9 kcal.g ${ }^{-1}$ de lipídio ${ }^{5}$.

\subsection{Estimativa do teor de cálcio das formulações sem fortificação}

Estimou-se o conteúdo de cálcio (análise indireta) das formulações sem a fortificação com o pó da casca de ovo, por meio de tabela de composição de alimentos ${ }^{10}$, considerando-se a composição química dos alimentos in natura. Foi determinado o índice de rendimento para cada formulação (relação entre o peso do alimento processado e o peso do alimento $\mathrm{cru})^{24}$, visando à correção dos valores obtidos em ingredientes crus para valores correspondentes em alimentos processados.

\subsection{Análise de cálcio do pó da casca de ovo e das formulações fortificadas}

O teor de cálcio do pó da casca de ovo foi determinado mediante método volumétrico, em triplicata, após digestão das amostras com ácido clorídrico e titulação com solução de EDTA, usando calceína como indicador ${ }^{3}$. As formulações fortificadas foram submetidas, em três replicatas, à análise do teor de cálcio por absorção atômica (espectrofotômetro marca VARIAN, modelo SpectrAA-200) ${ }^{1}$, usando-se lâmpada de catodo oco para o cálcio e os seguintes parâmetros instrumentais: comprimento de onda - 422,7 nm; corrente da lâmpada $10 \mathrm{~mA}$; largura da fenda $-0,5 \mathrm{~nm}$.

\subsection{Análise comparativa do teor de cálcio das formulaçóes fortificadas}

Para analisar a diferença existente entre a concentração média de cálcio de cada alimento fortificado (número de amostra e desvio-padrão conhecidos) em relação ao mesmo alimento sem fortificação, foi aplicado o teste $t$ de Student com nível de significância de $1 \%$, considerando-se o valor de cálcio do alimento sem fortificação (análise indireta) como único e constante ${ }^{25}$.

Para estimar a contribuição nutricional das porções dos alimentos fortificados para o aporte diário de cálcio, os teores do mineral contidos em cada porção das formulações fortificadas foram comparados com as referências nutricionais (ingestão adequada - AI) estabelecidas para o cálcio ${ }^{21}$.

\section{Resultados e discussão}

O pó da casca de ovo continha $37,4 \pm 0,2$ g. $100 \mathrm{~g}^{-1}$ ( $1,1 \%$ de umidade) de cálcio, tendo a casca de ovo in natura 32,2 g. 100 g-1 (15,1\% de umidade). Essa alta concentração de cálcio no pó era esperada, uma vez que a casca de ovo é composta essencialmente de carbonato de cálcio, sendo esse teor compatível com o relatado na literatura, de 36,8 g. $100 \mathrm{~g}^{-1}{ }^{16}$.

As concentrações em nutrientes e teor energético das formulações fortificadas com o pó da casca de ovo estão descritos na Tabela 2. Em relação aos macronutrientes, as formulações apresentaram teores mais elevados de carboidratos, de aproximadamente $50 \pm 10 \%$ (exceto arroz cozido), e baixas concentrações de proteína. Observa-se que o arroz cozido é o alimento com menor valor energético (150 kcal.100 $\mathrm{g}^{-1}$ ), e o biscoito de polvilho o que apresenta maior teor energético (cerca de $510 \mathrm{kcal} .100 \mathrm{~g}^{-1}$ ) e lipídico.

Os alimentos fortificados com o pó da casca de ovo apresentaram teores de cálcio entre $111,5 \mathrm{mg}$ e 506,4 mg. $100 \mathrm{~g} \mathrm{~g}^{-1}$ (Tabela 2). Em termos de porções médias, esses alimentos contribuem com 14 a $32 \%$ da ingestão adequada (AI) de cálcio para indivíduos adultos (1000 mg.dia-1 $)^{21}$, e apresentam concentrações do nutriente significativamente maiores ( $\mathrm{p}<0,01$ ) que as formulações originais sem fortificação (Tabela 3). Sendo assim, os alimentos fortificados podem contribuir de modo significativo para o aporte adequado de cálcio, sobretudo em situações fisiológicas de maior demanda do mineral. A ingestão diária de duas porções desses alimentos, por exemplo, beiju e bolo simples, perfazem cerca de $40 \%$ da AI de cálcio para adolescentes (1300 mg) e idosos (1200 mg) $)^{21}$ (Tabela 3). No entanto, a ingestão das mesmas formulações sem a fortificação representa aproximadamente $10 \%$ da AI para os referidos grupos.

A Portaria n ${ }^{\circ}$ 27/98 da Secretaria de Vigilância Sanitária do Ministério da Saúde ${ }^{4}$ define como alimento fonte de vitaminas e minerais, "aquele com mínimo de $15 \%$ da Ingestão Diária Recomendada (IDR) de referência por 100 g de alimento sólido", e como alimento rico em vitaminas e minerais, "aquele com mínimo de 30\% da IDR de referência por $100 \mathrm{~g}$ de alimento sólido". Assim, as formulações beiju com margarina e bolo de fubá de milho podem ser classificadas como alimentos fonte de cálcio, e as demais, exceto o arroz cozido e o arroz doce, como alimentos ricos em cálcio (Tabela 2). Dentre esses,

Tabela 2. Composição centesimal, valor energético e conteúdo de cálcio ${ }^{1}$ de alimentos fortificados com o pó da casca de ovo.

\begin{tabular}{|c|c|c|c|c|c|c|c|}
\hline \multirow[b]{2}{*}{ Formulação } & \multicolumn{7}{|c|}{ Composição centesimal (g.100 g $\left.{ }^{-1}\right)$, energia (kcal.100 g $\left.{ }^{-1}\right)$ e cálcio (mg.100 g $\left.{ }^{-1}\right)$} \\
\hline & Umidade & Energia & Proteínas & Lipídios & Carboidratos totais & Cinzas & Cálcio \\
\hline Arroz cozido & 63,88 & 150,1 & 2,50 & 2,36 & 29,72 & 1,24 & 111,5 \\
\hline Arroz doce & 55,52 & 177,8 & 2,08 & 0,53 & 41,35 & 0,52 & 116,2 \\
\hline Beiju com margarina & 31,26 & 275,8 & 0,14 & 2,85 & 62,39 & 2,11 & 290,4 \\
\hline Biscoito de polvilho & 12,48 & 509,3 & 5,67 & 27,30 & 52,68 & 1,87 & 428,0 \\
\hline Biscoito frito doce & 11,90 & 448,9 & 1,96 & 20,92 & 63,19 & 0,90 & 374,6 \\
\hline Biscoito frito salgado & 19,04 & 427,2 & 2,32 & 23,38 & 51,88 & 2,34 & 439,3 \\
\hline Bolinho de chuva & 15,71 & 415,3 & 5,43 & 17,24 & 59,61 & 1,86 & 506,4 \\
\hline Bolo de fubá de milho & 32,90 & 349,9 & 5,70 & 12,94 & 53,08 & 1,54 & 227,9 \\
\hline Bolo simples & 31,22 & 306,9 & 6,79 & 8,11 & 51,70 & 1,98 & 394,3 \\
\hline Farofa de ovo e banana & 31,77 & 301,7 & 2,73 & 8,69 & 53,15 & 2,46 & 303,8 \\
\hline
\end{tabular}

${ }^{1}$ Dados correspondem à média de três repetições/amostra. 
Tabela 3. Teor de cálcio (por porção) dos alimentos fortificados com o pó da casca de ovo em relação aos alimentos não-fortificados e às referências nutricionais (Ingestão Adequada - AI) para adolescente, adulto e idoso.

\begin{tabular}{|c|c|c|c|c|c|c|}
\hline \multirow[b]{3}{*}{ Formulação } & \multirow[b]{3}{*}{ Porção (g) } & \multirow{3}{*}{$\begin{array}{l}\text { Alimento não-fortificado } \\
\text { cálcio (mg/porção) }\end{array}$} & \multicolumn{4}{|c|}{ Alimento fortificado } \\
\hline & & & \multirow[b]{2}{*}{ cálcio (mg/porção) } & \multicolumn{3}{|c|}{ Porcentagem em relação à $\mathrm{AI}^{1}$} \\
\hline & & & & Adolescente & Adulto & Idoso \\
\hline Arroz cozido & 160 & 7,4 & $178,4^{*}$ & 14 & 18 & 15 \\
\hline Beiju com margarina & 70 & 4,1 & $203,3^{*}$ & 16 & 20 & 17 \\
\hline Biscoito de polvilho & 45 & 62,0 & $192,6^{*}$ & 15 & 19 & 16 \\
\hline Biscoito frito doce & 55 & 10,4 & $206,0^{*}$ & 16 & 21 & 17 \\
\hline Bolinho de chuva & 60 & 137,0 & $303,8^{*}$ & 23 & 30 & 25 \\
\hline Bolo de fubá de milho & 115 & 116,9 & $262,1^{*}$ & 20 & 26 & 22 \\
\hline Bolo simples & 80 & 151,0 & $315,4^{*}$ & 24 & 32 & 26 \\
\hline Farofa de ovo e banana & 45 & 17,5 & $136,7^{*}$ & 10 & 14 & 11 \\
\hline
\end{tabular}

${ }^{1} \mathrm{AI}$ para adolescente $=1300 \mathrm{mg}_{\text {dia }}{ }^{1}$; adulto $=1000 \mathrm{mg}$ dia $^{-1} ;$ idoso $=1200 \mathrm{mg} \cdot$ dia $^{-121}$; e ${ }^{*}$ Valores diferem significativamente dos teores de cálcio dos alimentos não-fortificados $($ Teste $t$, $\mathrm{p}<0,01)$.

destacam-se o biscoito de polvilho, o biscoito frito salgado, o bolinho de chuva e o bolo simples, sendo que os três últimos não continham alimentos fonte de cálcio em suas formulações originais (Tabela 1).

Os teores de cálcio das formulações arroz cozido e arroz doce não foram significativos em relação à AI dos grupos considerados. No caso do arroz cozido, que é em geral consumido em refeições contendo alimentos fonte de ferro, quantidades mais elevadas de cálcio não são recomendáveis, pois poderiam eventualmente inibir a biodisponibilidade do primeiro ${ }^{14}$. Já as formulações classificadas como fonte de cálcio ou ricas no mineral são consumidas em lanches, nos quais não há, geralmente, fontes alimentares de ferro.

No guia alimentar do Ministério da Saúde para a população brasileira ${ }^{19}$, está preconizada a ingestão de três porções diárias de produtos lácteos. Contudo, produtos lácteos não fazem parte da dieta habitual de adolescentes, adultos e idosos, em proporções suficientes para alcançar essas recomendações ${ }^{13,18,20,30}$. No caso de adolescentes e idosos, seriam necessárias pelo menos cinco porções diárias de leite e derivados para se atingir a $\mathrm{AI}^{21}$, o que acarretaria desequilíbrio na ingestão de macronutrientes, em razão da excessiva ingestão protéica que, por sua vez, poderia induzir a um aumento na excreção renal de cálcio ${ }^{8}$. Assim, a ingestão de alimentos de consumo habitual enriquecidos com o pó da casca de ovo pode contribuir para a ingestão adequada de cálcio sem comprometer a qualidade nutricional da dieta. Por exemplo, o consumo de somente duas porções de leite ( 1 porção $=200 \mathrm{~mL}$ ) ou derivados e de duas porções dos alimentos fortificados ricos em cálcio perfaz aproximadamente $100 \%$ da AI de cálcio para indivíduos de diferentes grupos etários, incluindo adolescentes e idosos ${ }^{10,21}$.

Desse modo, é recomendável o uso do pó da casca de ovo para fortificar alimentos consumidos habitualmente por diferentes grupos de indivíduos, pois, além de ser uma fonte de cálcio de boa qualidade nutricional, é um produto de fácil aquisição, de preparo simples e de baixo custo.

\section{Conclusões}

- A fortificação de alimentos com o pó da casca de ovo, nas proporções usadas no presente estudo, elevou o teor de cálcio das formulações testadas, resultando, na maioria, em alimentos ricos no mineral;

- Alimentos de consumo habitual fortificados com o pó da casca de ovo podem contribuir de forma significativa para a ingestão adequada de cálcio; e

- O pó da casca de ovo é uma fonte de cálcio viável e pode contribuir na prevenção de deficiência de cálcio, em especial na redução do risco de osteoporose, em indivíduos de diferentes grupos etários e extratos sociais.

\section{Agradecimentos}

Os autores agradecem à Pró-Reitoria de Assuntos da Comunidade Universitária pela concessão de bolsa-estágio e à Fundação de Apoio à Pesquisa (Funape) da Universidade Federal de Goiás pelo apoio financeiro à pesquisa.

\section{Referências bibliográficas}

1. ASSOCIATION OF OFFICIAL ANALYTICAL CHEMISTS (USA). Official Methods of Analysis. 15. ed. Arlington: AOAC, 1990. v. $1.684 \mathrm{p}$.

2. BLIGH, E. G.; DYER, W. J. A rapid method of total lipid extraction and purification. Canadian Journal of Biochemistry and Physiology, Ottawa, v. 37, n. 8, p. 911-917, 1959.

3. BRASIL. Ministério da Agricultura. Secretaria Nacional de Defesa Agropecuária. Análise de corretivos, fertilizantes e inoculantes: métodos oficiais. Brasília: LANARV, 1988. 104 p.

4. BRASIL. Ministério da Saúde. Secretaria de Vigilância Sanitária. Portaria $\mathbf{n}^{\circ} \mathbf{2 7}$, de $\mathbf{1 3}$ de janeiro de 1998. Aprova o regulamento técnico referente à informação nutricional complementar. Disponível em: <http://www.anvisa.gov.br/e-legis/> . Acesso em: 2 jul. 2005.

5. BRASIL. Agência Nacional de Vigilância Sanitária. Resolução $\mathbf{R D C}^{\circ}{ }^{\mathbf{9 4}}$, de 01 de novembro de 2000. Aprova o regulamento técnico para rotulagem obrigatória de alimentos e bebidas embalados. Disponível em: <http://www.anvisa.gov.br/e-legis/>. Acesso em: 3 jul. 2005.

6. COBAYASHI, F. Cálcio: seu papel na nutrição e saúde. Compacta Nutrição, São Paulo, v. 5, n. 2, p. 2-18, 2004. 
7. FLEMING, K. H.; HEIMBACH, J. T. Consumption of calcium in the U.S.: food sources and intake levels. Journal of Nutrition, Bethesda, v. 124, n. 8 (suppl.), p. 1426S-1430S, 1994.

8. GUÉGUEN, L.; POINTILLART, A. The bioavailability of dietary calcium. Journal of the American College of Nutrition, New York, v. 19, n. 2, p. 119S-136S, 2000.

9. INSTITUTO ADOLFO LUTZ. Normas analíticas do Instituto Adolfo Lutz: métodos químicos e físicos para análise de alimentos. 3. ed. São Paulo: IAL, 1985. 533 p.

10. INSTITUTO BRASILEIRO DE GEOGRAFIA E ESTATÍSTICA. Estudo Nacional de Despesa Familiar. Tabela de composição de alimentos. 5. ed. Rio de Janeiro: IBGE, 1999. 137 p.

11. INTERNATIONAL COMMISSION ON MICROBIOLOGICAL SPECIFICATION FOR FOODS. Ecologia microbiana de los alimentos 1: factores que afectam a la supervivencia de los microorganismos en los alimentos. España: Acribia, 1980.

12. LANZILLOTTI, H. S. et al. Osteoporose em mulheres na pósmenopausa, cálcio dietético e outros fatores de risco. Revista de Nutrição, Campinas, v. 16, n. 2, p. 181-193, 2003.

13. LERNER, B. R. et al. O cálcio consumido por adolescentes de escolas públicas de Osasco, São Paulo. Revista de Nutrição, Campinas, v. 13, n. 1, p. 57-63, 2000.

14. LYNCH, S. R. The effect of calcium on iron absorption. Nutrition Research Reviews, Cambridge, v. 13, n. 2, p. 141-158, 2000.

15. LUFT, N. et al. Efeito do cálcio da casca de ovo na mineralização óssea em ratos. In: CONGRESSO NACIONAL DA SOCIEDADE BRASILEIRA DE ALIMENTAÇÃO E NUTRIÇÃO, 8., 2005, São Paulo. Anais... São Paulo: Sociedade Brasileira de Alimentação e Nutrição, 2005. p. 328.

16. LUFT, N. et al. Teor de cálcio e qualidade microbiológica da farinha da casca de ovo. In: CONGRESSO NACIONAL DA SOCIEDADE BRASILEIRA DE ALIMENTAÇÃO E NUTRIÇÃO, 8., 2005, São Paulo. Anais... São Paulo: Sociedade Brasileira de Alimentação e Nutrição, 2005. p. 351.

17. MAIA, C. M.; SERAFINI, A. B.; NAVES, M. M. V. Pó da casca de ovo como fonte alternativa de cálcio: avaliação microbiológica e uso do APPCC na padronização do modo de preparo. In: CONGRESSO BRASILEIRO DE NUTRIÇÃO, 17., 2002, Porto Alegre. Anais... Porto Alegre: Associação Brasileira de Nutrição, 2002. p. 157.

18. MANTOANElli, G.; VITAlle, M. S. S.; AMANCIO, O. M. S. Amenorréia e osteoporose em adolescentes atletas. Revista de Nutrição, Campinas, v. 15, n. 3, p. 319-332, 2002.
19. MINISTÉRIO DA SAÚDE (Brasil). Guia alimentar para a população brasileira: promovendo a alimentação saudável. Brasília, 2006. 210 p. (Série A, Normas e Manuais Técnicos).

20. MONGE-ROJAS, R.; NUNEZ, H. P. Dietary calcium intake by a group of 13 18- year-old Costa Rican teenagers. Archivos Latinoamericanos de Nutrición, Caracas, v. 51, n. 2, p. 127-131, 2001.

21. NATIONAL ACADEMY OF SCIENCES (USA). Dietary reference intake for calcium, phosphorus, magnesium, vitamin $D$ and fluoride. Washington, DC: National Academy, 1997. 448 p.

22. NAVES, M. M. V. et al. Culinária goiana: valor nutritivo de pratos tradicionais. Goiânia: Kelps, 2004. 82 p.

23. PRENTICE, A. Calcium in pregnancy and lactation. Annual Review of Nutrition, Palo Alto, v. 20, p. 249-272, 2000.

24. SILVA, M. R. et al. Composição em nutrientes e valor energético de pratos tradicionais de Goiás, Brasil. Ciência e Tecnologia de Alimentos, Campinas, v. 23 (supl.), p. 140-145, 2003.

25. SILVA, M. R.; NAVES, M. M. V.; OLIVEIRA, A. G. Composição química de pratos à base de milho: comparação entre dados laboratoriais e de tabelas. Revista do Instituto Adolfo Lutz, São Paulo, v. 63, n. 2, p. 193-199, 2004.

26. SILVA JUNIOR, E. A. Manual de controle higiênico-sanitário em serviços de alimentação, 6. ed. São Paulo: Varela, 2005.

27. SOUSA, I. A. et al. Biodisponibilidade do cálcio da casca de ovo em rações à base de caseína e da mistura arroz-feijão. In: CONGRESSO NACIONAL DA SOCIEDADE BRASILEIRA DE ALIMENTAÇÃO E NUTRIÇÃO, 6., 2001, Florianópolis. Anais... Florianópolis: Sociedade Brasileira de Alimentação e Nutrição, 2001. p. 121.

28. WEAVER, C. M.; HEANEY, R. P. Cálcio. In: SHILS, M. E. et al. (Ed.). Tratado de nutrição moderna na saúde e na doença. 9. ed. Rio de Janeiro: Manole, 2003. cap. 7, p.153-168. (Tradução de: Modern nutrition in health and disease).

29. WEAVER, C. M.; PLAWECKI, K. L. Dietary calcium: adequacy of a vegetarian diet. American Journal of Clinical Nutrition, New York, v. 59, n. 5 (suppl.), p. 1238S-1241S, 1994.

30. WEINSIER, R. L.; KRUMDIECK, C. L. Dairy foods and bone health: examination of the evidence. American Journal of Clinical Nutrition, New York, v. 72, n. 3, p. 681-689, 2000. 